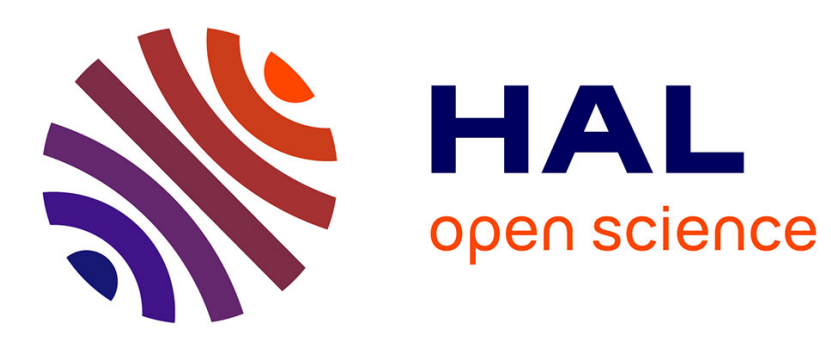

\title{
In-body Network Biomedical Applications: from Modeling to Experimentation
}

Valeria Loscrì, Ladislau Matekovits, Ildiko Peter, Anna Maria Vegni

\section{To cite this version:}

Valeria Loscrì, Ladislau Matekovits, Ildiko Peter, Anna Maria Vegni. In-body Network Biomedical Applications: from Modeling to Experimentation. IEEE Transactions on NanoBioscience, 2016, 10.1109/TNB.2016.2521386 . hal-01262020

\section{HAL Id: hal-01262020 \\ https://inria.hal.science/hal-01262020}

Submitted on 26 Jan 2016

HAL is a multi-disciplinary open access archive for the deposit and dissemination of scientific research documents, whether they are published or not. The documents may come from teaching and research institutions in France or abroad, or from public or private research centers.
L'archive ouverte pluridisciplinaire HAL, est destinée au dépôt et à la diffusion de documents scientifiques de niveau recherche, publiés ou non, émanant des établissements d'enseignement et de recherche français ou étrangers, des laboratoires publics ou privés. 


\title{
In-body Network Biomedical Applications: from Modeling to Experimentation
}

\author{
Valeria Loscrí Member, IEEE, Ladislau Matekovits Senior Member, IEEE, Ildiko Peter, and Anna Maria Vegni \\ Member, IEEE
}

\begin{abstract}
Innovative diagnostic approaches and therapies are more and more based on the use of injections or oral delivery of nanoparticle sized substances. For a better understanding of the overall phenomena, aiming to facilitate a safe application at large scale, the development of accurate models and analysis techniques are required. These techniques take into consideration different aspects of the overall process: accurate numerical modeling of the different phases of the nanoparticles in the body, and knowledge of the local environment, that can be varying very fast within a short-range in the body itself. Such aspects should be taken into account to correctly predict the amount of drug and its timely release for the specific disease. Deep and accurate analysis of the interaction between the nanoparticles and the biological fluid where the nanoparticles are immersed is mandatory for an efficient correlation of all these aspects.

Because of their biocompatibility, in this paper, we focus our attention on systems of Titanium (Ti), and its oxide (e.g., $\mathrm{TiO}_{2}$ ), given their specific features in terms of density, lack of cytotoxic effects, etc. Specifically, we present the study and design of an in-body system by characterizing each of the emission, diffusion, and reception processes with a proper realistic model. The theoretical investigation is further supported by experimental study of the morphology and other important characteristics (e.g., the $\mathrm{pH}$ of the particles, and thermal stability) of $\mathrm{TiO}_{2}$ systems when immersed in a Ringer solution, in order to derive important information related to their potential toxicity inside the human body.
\end{abstract}

Keywords-Nanomedicine, $\mathrm{TiO}_{2}$ particles, $\mathrm{THz}$ band channel capacity

\section{INTRODUCTION}

Nanotechnology is a challenging new research area that finds applications in several areas of modern life i.e., from biomedical to military, and from environmental to the industrial fields. For instance, in nanomedicine, the use of nanodevices (i.e. nanorobots and nanosensors, opportunely functionalized with medicament) are intended to both diagnostic and therapeutic purposes [1]-[3] by enhancing their effectiveness, as well as increasing their tolerability in the body by interfering at a minimum level with the immune system [4]. Novel nanomedicine applications arise to the term "theragnostics", which is intended as a treatment

\footnotetext{
Valeria Loscrí is with INRIA, Lille Nord-Europe, France. Email: valeria.loscri@inria.fr

Ladislau Matekovits is with the Department of Electronics and Telecommunications, Politecnico di Torino, 24, C.so Duca degli Abruzzi, 10129 Torino, Italy, and with the Macquarie University, Sydney 2109, NSW, Australia. Email: ladislau.matekovits@polito.it

Ildiko Peter is with the Department of Applied Science and Technology, Politecnico di Torino, 24, C.so Duca degli Abruzzi, 10129 Torino, Italy. Email: ildiko.peter@polito.it

Anna Maria Vegni is with Rome TRE University, Department of Engineering, COMLAB Telecommunication lab., Via Vito Volterra, 62 - 00146, Rome, Italy. Email: annamaria.vegni@uniroma3.it (corresponding author)
}

strategy combining therapeutics with diagnostics. Basically, theragnostics includes a wide range of areas, including personalized medicine, and pharmacogenomics, in order to develop efficient new targeted therapies.

Different types of nanoparticles can be exploited for nanomedicine applications, such as gold and silver nanoparticles, titanium dioxide $\mathrm{TiO}_{2}$ nanoparticles, dendrimers, Carbon Nano-Tubes (CNTs), etc. [5]. The use of nanoparticles for biomedical and sensing applications is mainly as carriers for localized Drug Delivery Systems (DDSs) [6]-[8]. The aim is to provide a controlled drug concentration in a given location, where the medication is needed [1]. Alternatively, in the case of diagnosis, the aim is to provide information about in-body conditions, by opportunistically measuring specific parameters and/or chemical concentrations, etc. [2] altering as less as possible the local environment. Particularly, colloidal silver has been used as an antibacterial agent by weakening DNA replication and inactivating proteins, while the low toxicity in biological systems of gold does not allow its efficient use for antibiotic therapy [9]. In [10], the authors use gold nanoparticles for diagnostic and drug delivery applications by exploiting chitosan, with the dual purpose of $(i)$ acting as a reducing agent in the synthesis of gold nanoparticles, and also (ii) promoting the penetration and uptaking the peptide hormone insulin across the mucosa.

The use of $\mathrm{TiO}_{2}, \mathrm{SiO}_{2}$ and $\mathrm{TiO}_{2} / \mathrm{SiO}_{2}$ obtained by different analytical techniques, and in different arrangements, is presented in [11]-[14] for controlled delivery of drugs for therapeutic purpose. The authors highlight also the positive effect of such particles, since they act against bacterial infection too and facilitate the osteoblast cell adhesion and growth. Additionally, particles with high surface area, large pore volume and proper pore size are important to guarantee an excellent adsorption capacity in the therapeutic system.

In the context of body area networks [15], nanoparticulate DDSs are therapeutic methods that use nanoparticles to achieve diagnostic effects at the exact time, drug concentration level, and location in the body, while minimizing the effects on other healthy locations. In both DDS and diagnostic applications, common methods adopted include inhalation, intravenous and intramuscular injection, as well as ingestion [16]. In all these methods, the most important thing is to characterize the interaction between the in-body solution and the specific nanoparticles.

The Molecular Communication (MC) [17]-[19] paradigm allows the analysis of a nanoparticulate DDS from the information theory perspective, as a tool to study nanoscale biological and medical systems [20], [21]. In [21] the pharmacokinetics of a DDS is analytically modeled through the abstraction of MC paradigm. The authors consider physicochemical processes in the particle propagation, such as $(i)$ the advection process i.e., the transport of particles due to the 
blood velocity, (ii) the diffusion process i.e., the Brownian motion of particles, (iii) the absorption process i.e., the particle absorption through tissues surrounding the blood vessels, (iv) the reaction process i.e., the reaction between the particles and the blood, and (v) the adhesion process i.e., when biomolecules bind to the drug-loaded particles. Finally, in [22], Chahibi and Akyildiz model a drug delivery system affected by noise effects in the nanoparticulate scenario of the cardiovascular system.

In this paper, we refer to the MC paradigm in order to analyze main features of a DDS, where nanoparticles are emitted, diffuse and then are captured (received) in a biological fluid. The DDS can be modeled as an end-toend system, where the nanoparticle emission process can be assumed as a transmitted message, and the received message is the nanoparticle reception process. Furthermore, in order to provide completeness to the paper, we carry out experimental results on $\mathrm{TiO}_{2}$ nanoparticles flow, which has been immersed into a biological fluid. As it can be noticed, in this paper a multi-disciplinary approach has been followed for the DDS modeling. Specifically, we refer to our previous work [23], where a nanoparticulate system has been modeled through pharmacokinetics laws, and provide a more accurate end-to-end DDS model, together with extensive experimental results.

The contribution of this paper is twofold. First, we model a drug delivery system for nanomedicine applications, through the characterization of nanoparticles emission, diffusion, and reception processes, that are analyzed from the information theory perspective. We assume that a nanoparticle flow is introduced within a biological fluid, and it is free to diffuse until reaching a given position. The model linking the nanoparticle emission and reception is investigated as an end-to-end system, where the capacity is derived analytically in case of ideal, and non-ideal scenarios. Finally, we carry out an experimental analysis of specific nanoparticles (i.e., $\mathrm{TiO}_{2}$ nanoparticles) in a biologic-like fluidic medium, in order to infer main features as $\mathrm{pH}$ stability and diffraction patterns modification determined by mediumterm permanence in biological-like fluid or quantification of the activation energy in the given conditions. Some preliminary results of our investigations have been recently presented in [23] that have been further enriched here. In particular, $(i)$ the development of the reception process and (ii) additional experimental characterizations on the behavior of the considered $\mathrm{TiO}_{2}$ system in a biologic-like fluidic medium have been considered.

Notice that the choice of $\mathrm{TiO}_{2}$ particles is due to their benefits for biomedical applications. Indeed, among other existing systems, because of their low density, excellent corrosion resistance, lack of cytotoxic effects, and good mechanical properties, $\mathrm{Ti}$ and its compounds (i.e., $\mathrm{TiO}_{2}$ ) are well established materials for biomedical applications, as orthopedic and dental implants, cardiovascular stents, joint replacement [24]-[28]. The use of a controlled drug delivery of $\mathrm{TiO}_{2}$ particles could enlarge the potential applications in biomedical fields.

This paper is organized as follows. In Section II we present our general (and not specific to materials discussed later in the paper) nanoparticulate drug delivery model. We highlight the main processes that occur when a concentration of nanoparticles is introduced in what we name here "compartment" to indicate a closed volume containing a biological fluid. Specifically, we detail the laws characterizing the nanoparticle emission/ transmission ${ }^{1}$, diffusion, and reception processes, from the information theory perspective. At the end of this section we will be able to derive the capacity of the proposed system (both in case of ideal and non-ideal nanoparticle reception). In Section III we describe the details of the experiment we dealt in order to infer the main characteristics of the interactions between the particles and the biological fluid. Finally, conclusions are drawn at the end of the paper.

\section{ChARACTERIZATION OF THE IN-BODY PHASES}

As early described in [23], our proposed nanoparticulate model is based on pharmacokinetics laws, which provide a mathematical basis for analyzing how the human body reacts to the administration of "external substances" (i.e. a fluid with a given nanoparticle concentration). Pharmacokinetics considers the rate at which drug concentrations change in the body, and includes the kinetics of absorption, distribution, metabolism and excretion of a drug. Also, from [21], in a blood network, we can suppose that a nanoparticle flow propagates subject to the physicochemical processes such as absorption, reaction, adhesion, diffusion, and advection.

In this paper, we rely on one compartment pharmacokinetics model for oral drug administration. This is an empirical approach, where the compartment can represent a body/organ volume, and a compartment is modeled with a biological fluid (e.g. the Ringer solution as described in Section III) into which a flow of nanoparticles is added by a rapid single dose (emission process), diffuses in the body (diffusion process), and finally reaches a given location for therapeutic/diagnostic aim (reception process).

\section{A. Nanoparticle emission process}

When a flow of nanoparticles is injected in the biologic fluid, the nanoparticle concentration i.e., $C_{N P}(t)$, increases according to an uniform behavior in the "compartment", until reaching a maximum value. The analysis of this model relies on a simple mass balance compliant with the dissolution kinetics of $\mathrm{TiO}_{2}$ nanoparticles [29] that have been used in the experiments described in Section III.

The mass balance model that provides the nanoparticle transmission rate in the compartment model is expressed as

$$
r_{T x}(t)=\frac{d C_{N P}(t)}{d t}=k_{d} c_{i n}-k_{e} C_{N P}(t),
$$

where $C_{N P}(t)$ is the nanoparticle concentration behavior along time, $c_{i n}$ is the initial nanoparticle concentration, $k_{d}$ [min $\left.{ }^{-1}\right]$ and $k_{e}\left[\mathrm{~h}^{-1}\right]$ are the first-order dissolution and elimination rate constants, respectively.

The solution of (1) shows a faster increase in the concentration for higher values of dissolution constant, while it decreases very slowly due to typical value of elimination constant i.e., $k_{e}=\ln (2) / t_{1 / 2}$, where $t_{1 / 2}[\mathrm{~h}]$ is the elimination half-life, that is the time required for the concentration of the nanoparticles to reach half of its original value (i.e., $\left.t_{1 / 2}=12 \mathrm{~h}\right)$.

The behavior of nanoparticle concentration in the compartment reflects that of a pulse-based modulation

\footnotetext{
${ }^{1}$ In this paper, the terms emission and transmission are interchanged.
} 
scheme [30], where a transmitter nanomachine instantaneously releases a pulse of molecules [31]. A detailed description of the nanoparticle concentration behavior has previously been analysed in [23].

In a generic blood vessel, the nanoparticle transmission rate $r_{T x}(t)$ is linked to the nanoparticle delivery rate $r_{R x}(t)$ through the following expression:

$$
r_{R x}(t)=\int_{-\infty}^{\infty} r_{T x}(\tau) h(t, \tau) d \tau
$$

where $h(t, \tau)$ is the time-varying impulse response of the MC link that takes into account for physicochemical processes. The analytical solutions of (2) can be obtained through the Harmonic Transfer Matrix method. Due to the fluctuations in the blood flow, the impulse response of the system depends on the state of the blood flow (local environment) at the time of the injection. The analytical expression of the time-varying impulse response of a MC link depends on $(i)$ the apparent velocity, (ii) the effective diffusivity, and (iii) the reaction process.

Finally, from [21] we can derive the desired nanoparticle transmission rate $r_{T x}^{*}$, as

$$
r_{T x}^{*}(t)=\sum_{i=1}^{K} r_{T x, i}^{*} \cdot \operatorname{sinc}\left(\frac{t-i T_{s}}{T_{s}}\right),
$$

where $K$ is the number of samples, and $T_{s}$ is the sampling period that satisfies the Nyquist criterion i.e., $T_{s}<(2 B)^{-1}$, with $B$ the bandwidth of the time-varying impulse response of the system. Notice that the sampling period depends on the blood velocity and the characteristic time scale of the advection-diffusion process.

\section{B. Nanoparticle diffusion process}

The diffusion process is analysed by distinguishing two cases i.e., (i) the ideal, and (ii) non-ideal one. In the first scenario, the nanoparticle concentration is assumed as homogeneous and no nanoparticle is lost during the diffusion. On the other hand, in the non-ideal case a portion of nanoparticles is lost and cannot reach a destination point. As known, the reaction of the human immune system to the presence of nanoparticles can cause nanoparticle loss and errors in the diffusion and reception processes, respectively. A detailed study on nanoparticle loss in a nanoparticulate system is investigated in [32].

In an ideal case, the concentration is assumed homogeneous and the residence time attains a mean reference value. Unfortunately, in a real reactor the nanoparticle fluid spends a different time inside the reactor, depending on specific characteristics of the compartment. The ideal case that models the compartment can be represented through a bioreactor. A bioreactor is a reactor that utilizes either a living organism or one or more enzymes from a living organism to accomplish a certain chemical transformation.

For the non-ideal case, from (1), the nanoparticle transmission rate $r_{T x}(t)$ can be identified with the nanoparticle concentration flux $J_{T}(x, t)$ at the output of the tablet i.e., for $x=x_{T}$. The particle concentration flux $J_{T}(x, t)$ is dependent on the nanoparticle concentration gradient at time $t$ and position $x$ through the Fick's first law, as follows

$$
J_{T}(x, t)=-D \nabla C_{N P}(x, t),
$$

where $D\left[\mathrm{~cm}^{2} / \mathrm{s}\right]$ is the diffusion coefficient, assumed as a constant value for a given fluidic medium, and depending on the size and shape of nanoparticles, as well as the interaction with the solvent and viscosity of the solvent. Unfortunately, the Fick's first law works when applied to steady state systems, namely the concentration will keep constant both along the space and in the time. In our application, the concentration changes during the time, since nanoparticles diffuse along the space determining different levels of concentrations. For that, we need to consider the Fick's second law. In practice, the diffusion associated with the degradation kinetics yields to the following mass balance:

$$
\frac{\delta C_{N P}(x, t)}{\delta t}=D \frac{\delta^{2} C_{N P}(x, t)}{\delta x^{2}}+b^{\prime} \cdot C_{N P}(x, t),
$$

where $b^{\prime}$ is a kinetic rate constant, and $C_{N P}$ is the drug concentration as transmitted from the previous phase. Due to the thinness of the wall as compared to the overall compartment radius, we can use Cartesian coordinates as approximation instead of cylindrical coordinates.

We consider our compartment with a total volume $V$, and a flux $F$ going through the two reactors. Then, we can apply and solve the mass balances that follow directly from (5). The mass balance on the first reactor can be modeled as a first order differential equation, that is

$$
\frac{d C_{1}(t)}{d t}=-\left(\frac{2 F}{V}+k\right) C_{1}(t)+\left(\frac{2 F}{V}+k\right) C_{\text {input }}(t),
$$

with the following conditions

$$
C_{1}(t=0)=0, \quad C_{\text {input }}(t)=C_{N P} \cdot \delta(t) .
$$

This leads to the solution for $C_{1}(t)$ as

$$
C_{1}(t)=C_{N P} \cdot e^{-\left(\frac{k+1}{\tau}\right) t},
$$

where $\tau=\frac{V}{2 F}$. The quantity $\left(C_{N P}-C_{1}(t)\right)$ corresponds to the portion of nanoparticle concentration that will be directly eliminated by the body, without reaching the receiver.

As an analogy to (6), we can write the following mass balance equation:

$$
\frac{d C_{2}(t)}{d t}=-\left(\frac{2 F}{V}+k\right) C_{2}(t)+\left(\frac{2 F}{V}+k\right) \cdot e^{-\left(\frac{k+1}{\tau}\right) t},
$$

whose solution is

$$
C_{2}(t)=\frac{C_{N P} \cdot t}{\tau} \cdot e^{-\left(\frac{k+1}{\tau}\right) t},
$$

which represents the concentration of dissolved nanoparticles in the compartment.

As we have modeled a scenario as a fluidic medium where nanoparticles are free to move following the diffusion laws until reaching the target cells ${ }^{2}$, they are able to form chemical bonds with them. Thus, from (10), we can obtain the concentration of bonds i.e., $C_{b}(t)$, formed due to the dissolved nanoparticles bound with the receptors, which can be described by the following formula:

$$
C_{b}(t) \cdot u(t-\delta)= \begin{cases}N B \cdot\left[1-e^{\left(-\frac{t-\delta}{\tau}\right)}\right], & t>\delta \\ 0, & t \leq \delta\end{cases}
$$

\footnotetext{
${ }^{2}$ As an instance, a target cell is a tumor cell that has been previously detected.
} 
where $N B$ is the maximum number of bonds that can occur i.e., $N B=\max C_{2}(t), \tau[\mathrm{s}]$ is the delay necessary for the bond formation, $\delta[\mathrm{s}]$ is the start time of the reception process that is related to the diffusion delay of the nanoparticles, and $u(t)$ is the Heaviside step function.

\section{Nanoparticle reception process}

In the reception process, a nanoparticle is received when it forms chemical bonds with the receptors of a target cell, following the ligand-receptor bond phenomenon.

Not all nanoparticles can form bonds, but just those that reached the location of a target cell. Indeed, we can distinguish $(i)$ a portion of nanoparticles concentration able to reach a target cell (namely, the most resistant ${ }^{3}-\mathrm{MR}-$ nanoparticles), and (ii) the remaining part comprised of those nanoparticles that are directly eliminated by the elimination process, without reaching any target cell (namely, the less resistant-LR- nanoparticles) ${ }^{4}$. Indeed, the transmitter emits a flow of nanoparticles, which will diffuse in the medium. A few of them will have a most resistant behavior, and will be the dissolved nanoparticles (i.e., MR), while others will be the eliminated nanoparticles (i.e., LR). At the receiver side, only the MR nanoparticles will be received, while the LR nanoparticles will be eliminated.

In the capture process, we distinguish two cases for (i) ideal, and (ii) non-ideal channel. In the case of ideal channel, nanoparticles are emitted by the transmitter and are correctly bound by the receiver. This corresponds to no diffusion errors (e.g., no nanoparticle elimination), and no reception errors (e.g., no queuing delays at the receptors). On the other side, for the non-ideal channel, nanoparticles can be affected by diffusion errors (e.g., nanoparticles can be eliminated before reaching the receiver), or are not bound due to interfering nanoparticles (e.g., nanoparticles near the receptors that cause cross-talk and interference). As an instance, during the diffusion process, a few nanoparticles can be damaged by obstructions and other interfering molecules (e.g., the B-cells of the human immune system [33]), with the consequence that those nanoparticles are eliminated by the body before reaching target cells.

Let us model the transmitter as a discrete memory-less source that emits nanoparticles, all potentially able to diffuse in the volume and reach the target cells. By assuming the channel model as a binary symmetric channel, the transmitter is modeled as emitting a binary variable $X_{i}$ with $i=[1,2]$, whose values are $x_{1}=1$, and $x_{2}=0$, associated to one MR, and one LR nanoparticle, respectively. Then, the probabilities of emitting the symbols $x_{(1,2)}$ are

$$
P\left(X_{i}\right)=\left\{\begin{array}{l}
P\left(x_{1}\right)=p \\
P\left(x_{2}\right)=1-p .
\end{array}\right.
$$

The receiver can form binds only when a MR nanoparticle arrives. Then, the receiver can receive $(i)$ one or (ii) no nanoparticle. The received signal can be expressed as a binary variable $Y_{j}$ with $j=[1,2]$, whose values are $y_{1}=1$, and $y_{2}=0$, respectively associated to one bond, and no bond.

\footnotetext{
${ }^{3}$ The term "resistant" means the skill of nanoparticles to diffuse in the biological fluid, and then reach a target cell.

${ }^{4}$ Notice that also the MR nanoparticles will be eliminated by biodegradation process, after having absolved the drug delivery process.
}

The diffusion channel model is assumed as a binary symmetric channel. Also, we will distinguish between the ideal and non-ideal scenarios, respectively when the correct reception probability is maximum, and not.

In the case of ideal channel, we can calculate the following conditioned probabilities:

$$
\begin{aligned}
& P\left(y_{1} \mid x_{1}\right)=1, \quad P\left(y_{1} \mid x_{2}\right)=0, \\
& P\left(y_{2} \mid x_{1}\right)=0, \quad P\left(y_{2} \mid x_{2}\right)=1,
\end{aligned}
$$

from which we can obtain the mutual information in the ideal case (i.e., $I_{I}$ ), as

$I_{I}\left(X_{i}, Y_{j}\right)=\sum_{i} \sum_{j} P\left(x_{i}\right) P\left(y_{j} \mid x_{i}\right) \log \frac{P\left(y_{j} \mid x_{i}\right)}{\sum_{i} P\left(x_{i}\right) P\left(y_{j} \mid x_{i}\right)}$,

which simplified becomes

$$
I_{I}\left(X_{i}, Y_{j}\right)=p \log \frac{1}{p}+(1-p) \log \frac{1}{(1-p)},
$$

that represents the entropy at the transmitter. Finally, from (16), we can obtain the channel capacity in the case of ideal scenario as

$$
C_{I}=\max _{p}\left[p \log \frac{1}{p}+(1-p) \log \frac{1}{(1-p)}\right] .
$$

Notice that (17) depends on the probability $p$ of emitting one MR nanoparticle, whose behavior is intuitively decreasing when the nanoparticle concentration $C_{N P}(t)$ decreases as well. The expression of $p$ can be formulated following the behavior of a low-pass filter in the frequency domain, that is

$$
p(\omega)=\frac{1}{1+j \omega \tau},
$$

where $\omega=2 \pi f[\mathrm{~Hz}]$, and $\tau[\mathrm{ms}]$ is the time constant of the $\mathrm{RC}$ circuit. Notice that $\tau$ has lower values corresponding to higher probabilities of one MR emission, meaning that slower is the slope of $|p(\omega)|$, faster is the increase of the nanoparticle concentration due to the emission of MR nanoparticles.

In the time domain, the probability $p$ results:

$$
p(t)= \begin{cases}\geq(1-p), & \text { for } t \leq t_{1}, \\ <(1-p), & \text { for } t>t_{1}\end{cases}
$$

where $t_{1}$ is the time instant where the concentration of nanoparticles is maximum. Eq. (19) states that for $t \leq t_{1}$ (i.e., for increasing values of $C_{N P}(t)$ ), the probability of emitting an MR nanoparticle is greater than (or equal to) the probability of emitting a LR nanoparticle i.e., $(1-p)$, while for $t>t_{1}$ i.e., for decreasing values of $C_{N P}(t)$, the probability of emitting an MR nanoparticle results lower than that of emitting a LR nanoparticle. On the other side, the probability $(1-p)$ of emitting a LR nanoparticle is expected to increase when the overall nanoparticle concentration decreases, that is for $t>t_{1}$.

Still referring to the circuit theory, it follows that the behavior of $q=(1-p)$ can be modeled as the charge of the capacitor $C$ in $\mathrm{RC}$ circuit, and its analytic expression is

$$
q(\omega)=\frac{j \omega \tau}{1+j \omega \tau}
$$




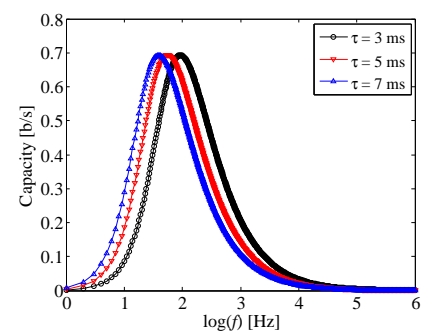

Fig. 1. Capacity $[\mathrm{b} / \mathrm{s}]$ of an ideal channel, for different values of frequency. Different curves correspond to different values of probability $p$, again depending on the time constant $\tau$.

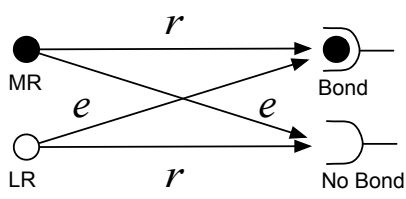

Fig. 2. Binary symmetric channel model for nanoparticle reception. A bond is formed between a MR nanoparticle and the receptor with probability $r$. Errors at the receiver can occur with probability $e$.

Referring back to (17), in the ideal scenario, the channel capacity can be expressed in terms of spectral efficiency $[\mathrm{b} / \mathrm{s} / \mathrm{Hz}]$, as shown in Fig. 1. This represents the information rate (i.e., the amount of nanoparticles in the time unit) that can be transmitted over a given bandwidth i.e., $\left[1,10^{6}\right] \mathrm{Hz}$. This is true since we have considered the system as a binary symmetric channel, when the nanoparticle emission/reception corresponds to bit emission/reception. Finally, the frequency spectrum considered in these analytic results ranges from $1 \mathrm{~Hz}$ to $1 \mathrm{MHz}$. Although the nanoparticles we are considering show size in the nanoscale level, corresponding to a frequency range in the $\mathrm{THz}$ band, we believe that the results in a lower frequency range could help the future development of nanoscale communication systems. Also, we do not consider a wider frequency range since such results up to $1 \mathrm{MHz}$ already show clearly the trend of the spectra efficiency as a function of the frequency, where the peak of capacity can be easily shifted to higher frequencies. An analogous consideration has been adopted in [34].

From Fig. 1, we observe that the three curves of capacity are obtained for different values of probability $p$. However, we notice the maximum values are the same, i.e. $C=0.69[\mathrm{~b} / \mathrm{s}]$, independently from the probability $p$, occurring for different values of frequency.

In the case of non-ideal channel, the system model can be represented as in Fig. 2, and the following conditioned probabilities hold:

$$
\begin{aligned}
& P\left(y_{1} \mid x_{1}\right)=r, \quad P\left(y_{1} \mid x_{2}\right)=e, \\
& P\left(y_{2} \mid x_{1}\right)=e, \quad P\left(y_{2} \mid x_{2}\right)=r,
\end{aligned}
$$

where $e=1-r$ i.e., with $0<e<1$, and $r$ i.e., with $0<r<1$, are the error and correct reception probability, respectively. This case corresponds to the ideal channel when $r=1$, and then, $e=0$. Notice that the error probability $e$ is when one of the following events occurs:

1) The transmitter emits one MR nanoparticle (i.e., $X_{i}=x_{1}$ ), but it is not able to be bound at the receptor, and no bond is formed (i.e., $Y_{i}=y_{2}$ );

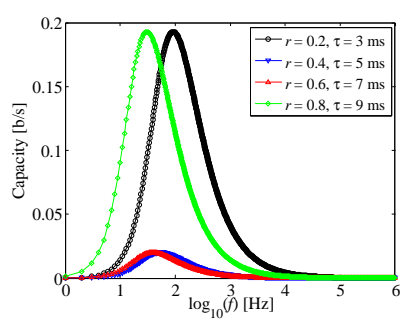

(a)

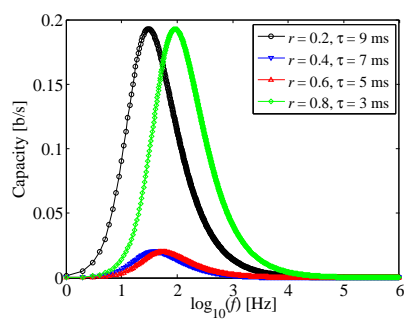

(b)
Fig. 3. Capacity $[\mathrm{b} / \mathrm{s}]$ of a non-ideal channel (i.e., $r \neq 1$ ). Different curves correspond to different values of probability $r$ and $\tau$.

2) The transmitter emits one LR nanoparticle (i.e., $X_{i}=$ $x_{2}$ ), and the receiver notifies one bond has been formed (i.e., $Y_{i}=y_{1}$ ).

The first event depends on the errors during the nanoparticle diffusion process; as an instance, one MR nanoparticle arrives at the receiver but can form no bond. On the other side, in the second event, one bond is formed even if the transmitter has emitted one LR nanoparticle, which is expected to be eliminated by the body, and then should never reach the receptor. However, it could happen that in the very near field of the receptor, one interfering nanoparticle could form a "fake" bond.

The expression of the mutual information for the non-ideal channel (i.e., $I_{N I}$ ) is

$$
\begin{aligned}
I_{N I}\left(X_{i}, Y_{i}\right) & =r \log r+(1-r) \log (1-r)+ \\
& -(1-p-r+2 r p) \log (1-p-r+2 r p)+ \\
& -(p-2 r p+r) \log (p+r-2 r p) .
\end{aligned}
$$

Let us pose $(p+r-2 r p)=\eta$, then (23) becomes

$$
\begin{aligned}
I_{N I}\left(X_{i}, Y_{i}\right) & =r \log r+(1-r) \log (1-r)+ \\
& -\eta \log \eta-(1-\eta) \log (1-\eta) .
\end{aligned}
$$

Notice that the meaning of $\eta$ can be represented also as:

$$
\eta=p\left(1-2 r+\frac{r}{p}\right)=p e+r q,
$$

where the first term pe represents the probability that one MR nanoparticle has been transmitted and an error has been notified at the receiver, while the term $r q$ is the probability that one LR nanoparticle has been transmitted and a correct reception has occurred. Such considerations are true since the probability of emitting one MR/LR nanoparticle is independent on the error occurring at the receiver, but it only depends on the channel noise and obstructions. Thus, $\eta$ represents the contributions of errors occurring in a nonideal channel.

Finally, from (24), we can obtain the channel capacity in the case of non-ideal scenario as

$$
\begin{aligned}
& C_{N I}= \max I_{N I}\left(X_{i}, Y_{i}\right)= \\
&=\max _{r, \eta}[r \log r+(1-r) \log (1-r) \\
&\quad-\eta \log \eta-(1-\eta) \log (1-\eta)] .
\end{aligned}
$$

Analogously to the case of ideal channel, the capacity still depends on the probability $p$ of emitting one MR nanoparticle, and moreover it depends also on the probabilities $r$ and $\eta$. 

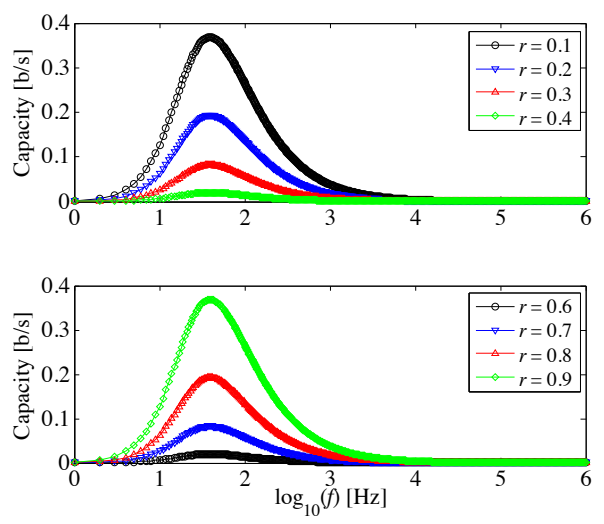

Fig. 4. Capacity [b/s] of a non-ideal channel for a fixed value of $\tau$ [ms] and different values of $r$.

Fig. 3 depict different curves of the channel capacity ${ }^{5}$, in the case of non-ideal channel, for different values of $r$ and $\tau$. In Fig. $3(a)$, we observe high capacity for highest and lowest values of the couple of correct reception probability $r$, and time constant $\tau$. This means that $(i)$ for $r=0.8$ and $\tau=$ $9 \mathrm{~ms}$ (i.e., green line) the correct reception probability is the highest but the lowest is the probability of MR nanoparticle emission, and (ii) for $r=0.2$ and $\tau=3 \mathrm{~ms}$ (i.e., black line) lowest is the correct reception probability but highest is the probability of MR nanoparticle emission. In these cases, the capacity results highest with frequency variations, due to different values of $\tau$. In contrast, for the other cases (see red and blue lines), the capacity is strongly reduced, still with frequency shifts.

Again, Fig. $3(b)$ shows similar behavior of capacity for different values of the couple $(r, \tau)$. This states that the magnitude of the capacity does not depends on the events of emitting one MR or LR nanoparticle (i.e., the value of $\tau$ ), but only on the channel behavior (i.e., the probability of correct/wrong reception). Indeed, given a fixed value of $\tau$, the higher values of capacity are obtained for $r \rightarrow 1$ for $r>0.5$, and for $r \rightarrow 0$ for $r<0.5$, while for $r=0.5$ the capacity is null. Fig. 4 depicts such a behavior of a non-ideal channel for $\tau=7 \mathrm{~ms}$. The highest capacity is $0.368[\mathrm{~b} / \mathrm{s}]$ given by $r=[0.1,0.9]$.

In the theoretical derivation of the emission, diffusion and reception processes, we characterize the spatio/temporal evolution of the nanoparticles without taking into account specific features of the nanoparticles and the fluid properties and interactions where they are immersed. This theoretical evaluation needs to be supported by a practical evaluation that allows the analysis of these features that would be taken into account as corrective factors in the theoretical model. In the next section we will show how to derive and evaluate the main factors characterizing the particles and the fluid as a whole system. The diffusion of the $\mathrm{TiO}_{2}$ particles is associated to the activation energy of the biological system.

\section{IN-BODY MODELING: EXPERIMENTAL EVALUATION AND CHARACTERIZATION}

In this section we will describe the details and features of the particles, and the solution considered to infer the main

\footnotetext{
${ }^{5}$ The channel capacity $[\mathrm{b} / \mathrm{s}]$ versus frequency represents the spectral efficiency $[\mathrm{b} / \mathrm{s} / \mathrm{Hz}]$.
}

features of the interaction between particles and body-like fluid solution. Specifically, we will study the morphology of the particles and any $\mathrm{pH}$ variations of the considered solutions. This analysis will allow the validation of the theoretical approach as derived in the previous section.

$\mathrm{TiO}_{2}$ (anatase, powder, 99.99\% purity; Sigma-Aldrich) and an aqueous solution of chlorides of sodium, potassium and calcium in the same concentrations as normal body fluids (Ringer solution, $7.5 \mathrm{ml}$ lactic acid, $5.85 \mathrm{~g} \mathrm{NaCl}, 300 \mathrm{ml}$ $\mathrm{H}_{2} \mathrm{O}$ di grade 2 purity, and $700 \mathrm{ml} \mathrm{H}_{2} \mathrm{O}$ and regulation of the $\mathrm{pH}$ to $6.9-7.0$ ) were used for the experiments. It is worth to notice that the particles we considered to deal our experiments are $\mu$-sized (i.e. 40 and $44 \mu \mathrm{m}$ ) because of the easier treatability and analysis of these particles. This solution is often used as a physiological solution for in vitro study of biomaterials due to its similarity to the body environment.

Two different $\mathrm{TiO}_{2}$ powders of dissimilar particle sizes, namely 40 and 44 micron respectively, have been selected for the experiments. Investigations on the metallic particles and their influence on the physiological solution has been carried out aiming to comprehend how these particles can influence the interactions between the biological system and the specific therapeutic nanostructure. In particular, structural and morphological observation by Scanning Electron Microscopy (SEM) and X-Ray diffraction, thermal analysis using Differential Scanning Calorimetry (DSC) and the metal ion release evaluation by $\mathrm{pH}$ measurements have been performed on the initial $\mathrm{TiO}_{2}$ particles and compared with data associated to samples after their permanence in simulated body fluids.

X-Ray diffraction patterns of the $\mathrm{TiO}_{2}$ powders, in the initial condition and after their 14 days permanence in the physiological solution are shown in Fig. 5. Both $\mathrm{X}$ Ray patterns related to the original powders show a strong diffraction peaks at $2 \theta=\left[25^{\circ}, 48^{\circ}\right]$ indicating the presence of the anatase phase. All peaks are in good agreement with the standard spectrum (JCPDS no 84-1286). As reported, the diffraction pattern peak intensity of the $\mathrm{TiO}_{2}$ increases with increasing particles size from $40 \mu \mathrm{m}$ to $44 \mu \mathrm{m}$. In terms of crystal structure, anatase, have $\mathrm{Ti}$ in an octahedral coordination with respect to the $\mathrm{O}$ atoms. In this crystal structure, $\mathrm{Ti}^{4+}$ is present as $\mathrm{TiO}_{6}$ octahedra and crystallize in a tetragonal unit cell. The development of monoclinic Titanium Hydrogen Oxide was observed as evidenced in Fig. 5 (dashed line ellipse), because of their permanence in the physiological solution.

The chemical bond established between the $\mathrm{TiO}_{2}$ particles and $\mathrm{O}$ and $\mathrm{H}$ atoms from the aqueous solution results in an un-stable structure, making the hydrophilic powders more reactive towards any possible chemical compound, and suitable for drug delivery with a correct ability of controlled release for a prolonged period.

In line with some literature data [35], [36], our DSC study indicates that at high temperature, phase transformation process occurs, and the exothermally and irreversibly transformation of anatase to rutile takes place, for the starting $\mathrm{TiO}_{2}$ powder with a significant reorganization of the structure. This transformation does not affect the considered in-body system, since it arises at higher temperature level that human body can be reals, as reported in the DSC thermograms of the $\mathrm{TiO}_{2}$ particles at $44 \mu \mathrm{m}$, in Fig. 6 


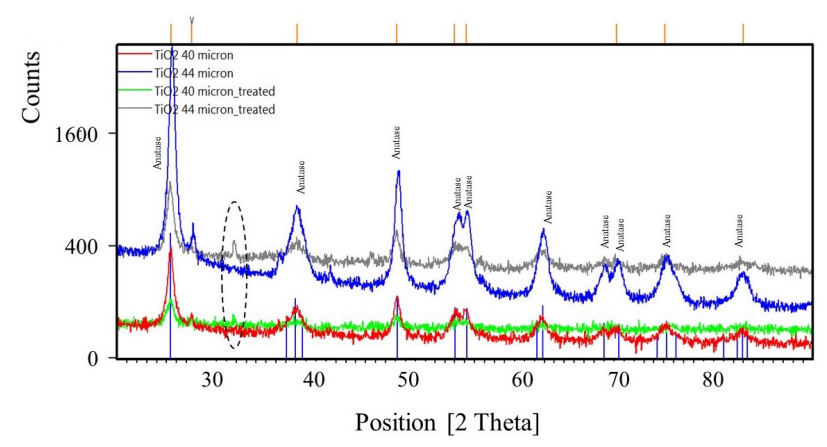

Fig. 5. X-Ray diffraction patterns for the $\mathrm{TiO}_{2}$ powders of both sizes (i.e., 40 and 44 micron): the initial powders, and after their permanence for 14 days in the physiological solution (labelled as "treated").

(a), but some information can be deduced concerning the necessary minimum energy to start a chemical reaction, or to reach a transition state in the case of drug transport and administration.

DSC studies were carried out to confirm the stability of the powders in the interest temperature range for the human body and to collect information about the $\mathrm{TiO}_{2}$ particles diffusion velocity in the biological environment by analyzing the activation energy of the system. The most important factors that influence the velocity of the reaction are (i) the nature and the concentration of the $\mathrm{TiO}_{2}$ and of the therapeutic particles, and (ii) the temperature. Proportionally to the increase of the reagents concentration the probability of the collisions and the velocity of the particles increases; higher surface contact between the particles favors higher reaction rate, while higher temperature leads obtaining more collisions between the particles and the velocity of the reaction become higher.

Calculations have been performed to estimate the kinetic parameter as activation energy and to qualitatively correlate it to the rate of the reaction. The activation energy is very low (i.e., $E_{a} \sim 11.65 \mathrm{~kJ} / \mathrm{mol}$ ) and, according to the metallic ion release measurements, no any chemical interaction between the $\mathrm{TiO}_{2}$ particles and the biological solution has been obtained. The activation energy will increase when anchoring the therapeutic particles to the $\mathrm{TiO}_{2}$ particles before immersing them in the biological fluid. There are ample space to the control of the reaction between $\mathrm{TiO}_{2}$ particles and the therapeutic particles in the preferred way according to the targeted application, i.e., selection of the proper drug and its quantity for the given treatement.

The relationship between activation energy, $E_{a}\left[\mathrm{~J} \mathrm{~mol}^{-1}\right]$, and the velocity of a chemical reaction is provided by Arrhenius equation i.e.,

$$
E_{a}=-R T \cdot \ln \frac{k}{A},
$$

where $k$ is a reaction rate constant (measured in $s^{-1}$ for first order reactions), $R$ is the universal gas constant (i.e., $\left.8.314472\left[\mathrm{~J} \mathrm{~K}^{-1} \mathrm{~mol}^{-1}\right]\right), A$ is the pre-exponential factor (measured in $\left[s^{-1}\right]$ for first order reactions), and $T$ is the temperature $[\mathrm{K}]$. The inverse exponential relationship between activation energy and the rate constant guarantees that the reactions with a very high activation energy will have a very low reaction rate. As activation energy decreases, the reaction rate increases. The activation energy is determined

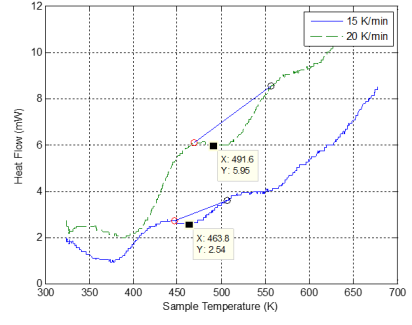

(a)

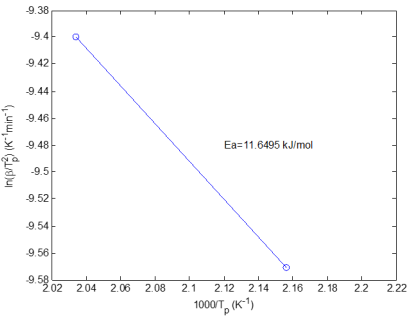

(b)
Fig. 6. (a) Heat flow curves of the $\mathrm{TiO}_{2}$ particles at $44 \mu \mathrm{m}$, obtained at two heating rates. $(b)$ Plot of $\ln \left(\beta / T_{p}\right)$ vs. $10^{3} / T_{p}$ for calculations of the activation energy based on the heating rate $\beta$ of the $\mathrm{TiO}_{2}$ particles.

by Kissinger's method i.e., with no precise knowledge of the mechanism of the reaction, the following equation can be used:

$$
\ln \left(\frac{\beta}{T_{p}^{2}}\right)=\left\{\ln \frac{A R}{E_{a}}+\ln \left[n(1-\alpha)^{n-1}\right]\right\}-\frac{E_{a}}{R T_{p}},
$$

where $T_{p}[\mathrm{~K}]$ is the temperature corresponding to the maximum reaction rate (peak), $\alpha$ is the conversion at $T_{p}, n$ is the reaction order, and $\beta$ is the heating rate expressed in $[\mathrm{K}$ $s^{-1}$ ]. Considering Kissinger's approximation, it follows:

$$
f\left(\alpha_{\max }\right)=n\left(1-\alpha_{\max }\right)^{n-1} \cong \text { const. }
$$

The activation energy can be determined from the plot of $\ln \left(\frac{\beta}{T_{p}^{2}}\right)$ versus $\frac{1}{T_{p}}$, as depicted in Fig. $6(b)$.

The $\mathrm{pH}$ of the solutions containing the $\mathrm{TiO}_{2}$ powders was measured at the pre-set time intervals during 14 days. The quantity of metal ions released in the physiological solution is negligible even after 14 days: the $\mathrm{pH}$ value of the solutions remains constant. The results obtained for the $\mathrm{pH}$ value and the weight loss fulfill the conditions required by the Standard ISO 10271/2011 concerning the evaluation of the corrosion resistance of the materials used in bio environment.

The particles shape and their surface reactivity influence the transport properties of other particles in the human environment, and thus affecting the adhesion kintetics of the therapeutic particles loaded on and their transfer to the favorite position. As illustrated in Fig. 7, the $\mathrm{TiO}_{2}$ particles show a controlled size distribution even if not perfectly regular as their size concerns. This aspect can be capitalized to improve the drug transport and their delivery efficiency as compared to the identical spherical shape particles. After their permanence in the physiological solution, some cluster development has been observed with increased surface area. This configuration highlights the positive effect coming from the permanence in the biological solution favoring the most efficient loading of the therapeutic particles obtaining higher attachment to the specific therapeutic particles as a vascular barrier.

\section{Conclusions}

Results of investigation on how nanoparticles behave in the human body for nanomedicine applications have been presented. The action is described by means of the modeling of the emission, diffusion and reception processes, which represent the overall end-to-end system from a transmitter to a receiver. In particular, the one compartment model for 


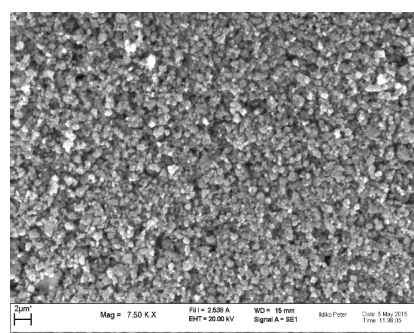

(a)

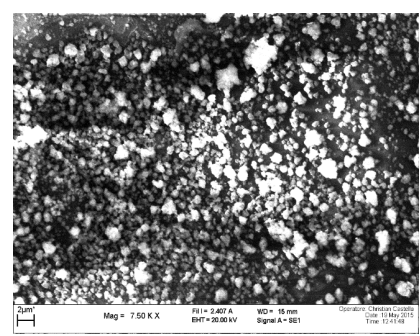

(c)

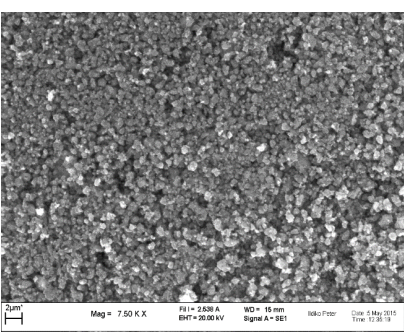

(b)

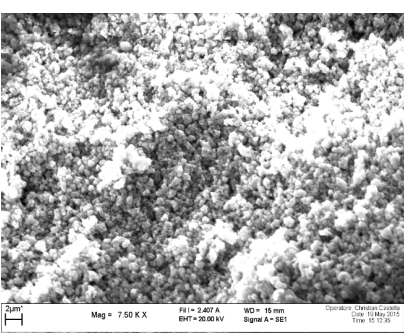

$(d)$
Fig. 7. SEM microstructure of $\mathrm{TiO}_{2}$ particles for different states and size i.e., (a) initial state and $40 \mu \mathrm{m}$ size, (b) initial state and $44 \mu \mathrm{m}$ size, (c) in solution and $40 \mu \mathrm{m}$ size, and $(d)$ in solution and $44 \mu \mathrm{m}$ size.

oral drug administration has been presented and discussed. From the characterization of the DDS channel we derived the capacity both in case of ideal and non-ideal scenario. Specifically, $\mathrm{TiO}_{2}$ particles in a biological-like fluid system as possible carrier to sustain drugs has further been considered.

Experimental results on their medium-time presence in biological-like fluids confirm their stability within the system, and put in evidence their capability to be used as carrier for drug delivery. The results of the investigation make evident the possibility of combining the biocompatibility of $\mathrm{TiO}_{2}$ to the drug delivery features and their use in biomedical field. The observed complete absence of any release of $\mathrm{Ti}$ ions in the physiological solution demonstrates, once again, a non-toxic character of the considered molecule. The particles with a high surface area show a higher tendency to be wetted by the aqueous biological solution and they can support more efficiently any drug loaded on their surfaces.

\section{REFERENCES}

[1] S. Svenson and R. K. Prud'homme, Multifunctional Nanoparticles for Drug Delivery Applications: Imaging, Targeting, and Delivery, Springer, Ed., 2012.

[2] R. A. Freitas, "What is nanomedicine?" Nanomedicine: nanotechnology, biology, and medicine, vol. 1, no. 1, pp. 2-9, March 2005.

[3] V. Loscrí and A. M. Vegni, "An acoustic communication technique of nanorobot swarms for nanomedicine applications," NanoBioscience, IEEE Transactions on, vol. PP, no. 99, pp. 1-1, 2015.

[4] - "On the affection of the human immune system on a nanoparticulate nanomedicine system," in Proceedings of the 9th International Conference on Body Area Networks - BODYNETs, 2014.

[5] N. Kumar, Handbook of Particulate Drug Delivery, A. S. Publishers, Ed., 2008.

[6] M. Hossen, K. Kajimoto, H. Akita, M. Hyodo, and H. Harashima, "Vascular-targeted nanotherapy for obesity: Unexpected passive targeting mechanism to obese fat for the enhancement of active drug delivery," J. Control. Release, vol. 163, pp. 101 - 110, 2012.
[7] D. Vllasaliu, C. Alexander, M. Garnett, M. Eaton, and S. Stolnik, "Fc-mediated transport of nanoparticles across airway epithelial cell layers," J. Control. Release, vol. 158, pp. 479-486, 2012.

[8] M. Femminella, G. Reali, and A. Vasilakos, "A molecular communications model for drug delivery," NanoBioscience, IEEE Transactions on, vol. 14, no. 8, pp. 935-945, Dec 2015.

[9] Y. Zhou, Y. Kong, S. Kundu, J. Cirillo, and H. Liang, "Antibacterial activities of gold and silver nanoparticles against escherichia coli and bacillus calmette-guerin," Journal of Nanobiotechnology, vol. 10, 2012.

[10] D. Bhumkar, H. Joshi, M. Sastry, and V. Pokharkar, "Chitosan reduced gold nanoparticles as novel carriers for transmucosal delivery of insulin," Pharmaceutical Research, vol. 24, 2007.

[11] Y.-Y. Song, F. Schmidt-Stein, S. Bauer, and P. Schmuki, "Amphiphilic TiO2 Nanotube Arrays: An Actively Controllable Drug Delivery System," Journal of the American Chemical Society, vol. 131, no. 12, pp. 4230-4232, 2009. [Online]. Available: http://dx.doi.org/10.1021/ja810130h

[12] K. C. Popat, M. Eltgroth, T. J. LaTempa, C. A. Grimes, and T. A. Desai, "Decreased staphylococcus epidermis adhesion and increased osteoblast functionality on antibiotic-loaded titania nanotubes," Biomaterials, vol. 28 , no. 32, pp. 4880 - 4888, 2007. [Online]. Available: http://www.sciencedirect.com/science/article/pii/S014296120700556X

[13] T. Wang, H. Jiang, L. Wan, Q. Zhao, T. Jiang, B. Wang, and S. Wang, "Potential application of functional porous $\mathrm{TiO} 2$ nanoparticles in light-controlled drug release and targeted drug delivery," Acta Biomaterialia, vol. 13, pp. 354 - 363, 2015. [Online]. Available: http://www.sciencedirect.com/science/article/pii/S1742706114005042

[14] M. Signoretto, E. Ghedini, V. Nichele, F. Pinna, V. Crocell, and G. Cerrato, "Effect of textural properties on the drug delivery behaviour of nanoporous $\mathrm{TiO} 2$ matrices ," Microporous and Mesoporous Materials , vol. 139, no. 13, pp. 189 - 196, 2011. [Online]. Available: http://www.sciencedirect.com/science/article/pii/S1387181110003719

[15] M. Chen, S. Gonzalez, A. Vasilakos, H. Cao, and V. C. Leung, "Body area networks: A survey," Mob. Netw. Appl., vol. 16, no. 2, pp. 171-193, Apr. 2011. [Online]. Available: http://dx.doi.org/10.1007/s11036-010-0260-8

[16] J. Cleland, A. Daugherty, and R. Mrsny, "Emerging protein delivery methods," Curr. Opin. Biotechnol., vol. 12, pp. 212-219, 2001.

[17] T. Nakano, T. Suda, Y. Okaie, M. Moore, and A. Vasilakos, "Molecular communication among biological nanomachines: A layered architecture and research issues," NanoBioscience, IEEE Transactions on, vol. 13, no. 3, pp. 169-197, Sept 2014.

[18] T. Nakano, M. Moore, F. Wei, A. Vasilakos, and J. Shuai, "Molecular communication and networking: Opportunities and challenges,' NanoBioscience, IEEE Transactions on, vol. 11, no. 2, pp. 135-148, June 2012.

[19] L. Felicetti, M. Femminella, G. Reali, T. Nakano, and A. Vasilakos, "Tcp-like molecular communications," Selected Areas in Communications, IEEE Journal on, vol. 32, no. 12, pp. 2354-2367, Dec 2014.

[20] Y. Chahibi, I. Akyildiz, and S. O. Song, "Antibody-based molecular communication for targeted drug delivery systems," in Engineering in Medicine and Biology Society (EMBC), 2014 36th Annual International Conference of the IEEE, Aug 2014, pp. 5707-5710.

[21] Y. Chahibi, M. Pierobon, and I. Akyildiz, "Pharmacokinetic modeling and biodistribution estimation through the molecular communication paradigm," Biomedical Engineering, IEEE Transactions on, vol. 62, no. 10, pp. 2410-2420, Oct 2015.

[22] Y. Chahibi and I. Akyildiz, "Molecular communication noise and capacity analysis for particulate drug delivery systems," Communications, IEEE Transactions on, vol. 62, no. 11, pp. 3891-3903, Noy 2014.

[23] V. Loscrí, L. Matekovits, I. Peter, and A. M. Vegni, "Modeling and experimental analysis of an in-body area nanonetwork," in Proceedings of the 10th EAI International Conference on Body Area Networks - BODYNETs, September 28-30 2015.

[24] M. Long and H. Rack, "Titanium alloys in total joint replacement a materials science perspective," Biomaterials, vol. 19, no. 18, pp. 1621 - 1639, 1998. 
[25] M. N. Helmus, D. F. Gibbons, and D. Cebon, "Biocompatibility: Meeting a key functional requirement of next-generation medical devices," Toxicologic Pathology, vol. 36, no. 1, pp. 70-80, 2008.

[26] E. Gultepe, D. Nagesha, S. Sridhar, and M. Amiji, "Nanoporous inorganic membranes or coatings for sustained drug delivery in implantable devices," Advanced Drug Delivery Reviews, vol. 62, no. 3, pp. $305-315,2010$.

[27] I. Peter and M. Rosso, "Study of Ti-Enriched CoCrMo alloy for dental application," Access, IEEE, vol. 3, pp. 73-80, 2015.

[28] I. Peter, M. Rosso, A. Toppi, I. Dan, and B. Ghiban, "Investigation on cobalt based alloy modified by titanium for dental application," Archives of Materials Science and Engineering, vol. 61, no. 2, pp. 62-68, June 2013.

[29] W. Utembe, K. Potgieter, A. B. Stefaniak, and M. Gulumian, "Dissolution kinetics of titanium dioxide nanoparticles: the observation of an unusual kinetic size effect," Particle and Fibre Toxicology, vol. 12, no. 11, pp. 1-12, 2015

[30] I. Llatser, E. Alarcon, and M. Pierobon, "Diffusion-based channel characterization in molecular nanonetworks," in Proc. of the 1st IEEE International Workshop on Molecular and Nano Scale Communication (MoNaCom), in conjunction with IEEE INFOCOM, Shanghai (China), April 2011.

[31] T. Nakano, Y. Okaie, and A. Vasilakos, "Transmission rate control for molecular communication among biological nanomachines," Selected Areas in Communications, IEEE Journal on, vol. 31, no. 12, pp. 835846, December 2013.

[32] V. Loscrí, A. M. Vegni, and G. Fortino, "On the interaction between a nanoparticulate system and the human body in body area nanonetworks," Micromachines, vol. 6, no. 9, p. 1213, 2015. [Online]. Available: http://www.mdpi.com/2072-666X/6/9/1213

[33] _ _ "On the interaction between a nanoparticulate system and the human body in body area nanonetworks," Micromachines, vol. 6, no. 9, p. 1213, 2015. [Online]. Available: http://www.mdpi.com/2072-666X/6/9/1213

[34] M. Pierobon and I. Akyildiz, "A physical end-to-end model for molecular communication in nanonetworks," IEEE Journal on Selected Areas in Communications, vol. 28, no. 4, pp. 602-611, 2010.

[35] Z. Wang and X. Deng, "A12O3 composite agent effects on phase transformation of nanometer $\mathrm{TiO} 2$ powder," Materials Science and Engineering: $B$, vol. 140, no. 12, pp. $109-113,2007$. [Online]. Available: http://www.sciencedirect.com/science/article/pii/S092151070700195X

[36] J. Huberty and H. Xu, "Kinetics study on phase transformation from titania polymorph brookite to rutile," Journal of Solid State Chemistry, vol. 181, no. 3, pp. 508-514, 2008.

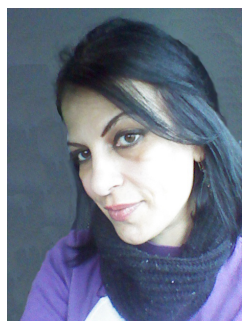

Valeria Loscrí is a permanent researcher of the FUN Team in Inria Lille - Nord Europe since the 1th October 2013. She got her Master degree in Computer Science and $\mathrm{PhD}$ in Systems Engineering and Computer Science in 2003 and 2007 respectively, both at University of Calabria (Italy). In 2006 she spent 6 months as visiting researcher at Rice University under the supervision of Prof. Knightly, where she worked on the MAC layer of wireless mesh networks. She authored more than 60 publications in journal, conferences, workshops and book chapters. She is involved in several programs and organization committees such as SWANSITY 2014, WiMob 2014, IDCS 2014, ICCCN 2012. Her research interests focus on performance evaluation, self-organizing systems, robotics networks, nanocommunications.

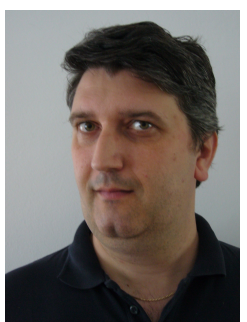

Ladislau Matekovits (M'94 - SM'11) received the degree in Electronic Engineering from Institutul Politehnic din Bucureşti, Romania and the Ph.D. in Electronic Engineering from Politecnico di Torino, Italy in 1992 and 1995 respectively. Since 1995 he has continuously been with the Electronics Department of the Politecnico di Torino where he has been appointed as Assistant Professor in 2002 and Associate Professor in 2014, respectively. Beginning July 1, 2009, for two years he has been a Marie Curie Fellow at Macquarie University, Sydney, NSW, Australia, where now he is Honorary Fellow. His main research activities concern computational electromagnetics, optimization techniques and active and passive metamaterials. He has delivered seminars on these topics all around the world: Europe, USA, Australia, China. He authored more than 250 publications in journals, conferences, workshops and book chapters. He is recipient of various awards including the 1998 URSI Young Scientist Award and the Best AP2000 Oral Paper on Antennas, ESA-EUREL Millenium Conference on Antennas \& Propagation. He has been Assistant Chairman and Publication Chairman of the European Microwave Week 2002 (Milan, Italy). He is member of the organizing committee of the International Conference on Electromagnetics in Advanced Applications (ICEAA) and of technical program committees of several conferences. He serves as Associated Editor of the IEEE Access, and reviewer for different journals.

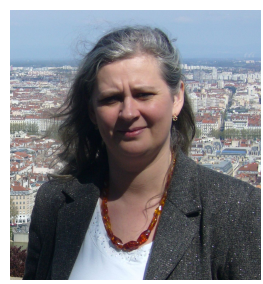

Ildiko Peter received the M.S. degree in Biochemistry (1993) and has obtained her $\mathrm{PhD}$ degree (2006) in Material Science and Engineering from Universitá degli Studi di Torino and Politecnico di Torino, Italy, respectively. Her main research interest is oriented to the development/characterization of different metallic alloys for automotive/aeronautical industries and biomedical applications, the synthesis and characterization of materials for advanced electromagnetic applications. She has co-authored more than 90 publications and holds two patents. In 2010 she has been a Visiting Researcher at Macquarie University, Sydney, NSW, Australia, has participated in various European and National projects. She is acting as reviewer for several Journals and since 2015 she serves as Associated Editor of the IEEE Access.

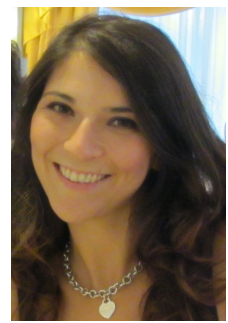

Anna Maria Vegni is non-tenured Assistant Professor in Telecommunications at the Department of Engineering of Roma Tre University, Rome, Italy. She received the Ph.D. degree in Biomedical Engineering, Electromagnetic and Telecommunications from Roma Tre University in 2010 and the Laurea Degree cum laude in Electronics Engineering in 2006. In 2009, she was a visiting researcher at Boston University (Boston, MA), under the supervision of Prof. Little, where she worked on vehicular networking. She authored around 70 publications in journals, conferences, workshops and book chapters. She is involved in several EU programs, and organization committees such as OWC ICC workshop 2016, and ACM NANOCOM 2016. She is a member of editorial board of JNCA and NANOCOMNETS (Elsevier) journals. Her research activity focuses on vehicular networking, indoor and outdoor localization, VLC, and nanocommunications. 